\title{
Stagnation and complex flow in ruptured cerebral aneurysms: a possible association with hemostatic pattern
}

\author{
Masanori Tsuji, MD, ${ }^{1}$ Tatsuya Ishikawa, MD, ${ }^{2}$ Fujimaro Ishida, MD, ${ }^{3}$ Kazuhiro Furukawa, MD, ${ }^{1}$ \\ Yoichi Miura, MD, ${ }^{4}$ Masato Shiba, MD, ${ }^{3}$ Takanori Sano, MD, ${ }^{1}$ Hiroshi Tanemura, MD, ${ }^{3}$ \\ Yasuyuki Umeda, MD, ${ }^{1}$ Shinichi Shimosaka, MD, ${ }^{3}$ and Hidenori Suzuki, MD'
}

\begin{abstract}
1Department of Neurosurgery, Mie University Graduate School of Medicine, Tsu, Mie; '2Department of Neurological Surgery, Research Institute for Brain and Blood Vessels, Akita, Akita; ${ }^{3}$ Department of Neurosurgery, Mie Chuo Medical Center, National Hospital Organization, Tsu, Mie; and ^Department of Neurosurgery, Suzuka Kaisei Hospital, Suzuka, Mie, Japan
\end{abstract}

\begin{abstract}
OBJECTIVE Histopathological examination has revealed that ruptured cerebral aneurysms have different hemostatic patterns depending on the location of the clot formation. In this study, the authors investigated whether the hemostatic patterns had specific hemodynamic features using computational fluid dynamics (CFD) analysis.
\end{abstract}

METHODS Twenty-six ruptured middle cerebral artery aneurysms were evaluated by 3D CT angiography and harvested at the time of clipping. The hemostatic patterns at the rupture points were assessed by means of histopathological examination, and morphological parameters were obtained. Transient analysis was performed, and wall shear stress-related hemodynamic parameters and invariant $Q$ (vortex core region) were calculated. The morphological and hemodynamic parameters were compared among the hemostatic patterns.

RESULTS Hematoxylin and eosin staining of the aneurysm wall showed 13 inside-pattern, 9 outside-pattern, and 4 other-pattern aneurysms. Three of the 26 aneurysms were excluded from further analysis, because their geometry models could not be generated due to low vascular CT values. Mann-Whitney U-tests showed that lower dome volume $(0.04$ $\mathrm{cm}^{3}$ vs $\left.0.12 \mathrm{~cm}^{3}, p=0.014\right)$, gradient oscillatory number ( 0.0234 vs $\left.0.0289, p=0.023\right)$, invariant $Q\left(-0.80110^{-2} / \mathrm{sec}^{2}\right.$ vs $\left.-0.12410^{-2} / \mathrm{sec}^{2}, p=0.045\right)$ and higher aneurysm formation indicator $(0.986$ vs $0.963, p=0.041)$ were significantly related to inside-pattern aneurysms when compared with outside-pattern aneurysms.

CONCLUSIONS Inside-pattern aneurysms may have simpler flow patterns and less flow stagnation than outside-pattern aneurysms. CFD may be useful to characterize the hemostatic pattern of ruptured cerebral aneurysms.

https://thejns.org/doi/abs/10.3171/2016.3.JNS152264

KEY WORDS computational fluid dynamics; hemostatic mechanism; ruptured aneurysm; histopathological examination; vascular disorders

$\mathrm{R}$ UPTURE of cerebral aneurysms causes subarachnoid hemorrhage, and survival of patients experiencing this devastating phenomenon requires immediate cessation of aneurysmal bleeding. Although a significant number of ruptured cerebral aneurysms seal and form a hemostatic plug, the hemostatic mechanism has not been fully clarified. Ruptured cerebral aneurysms have been histopathologically classified largely into 2 hemostatic patterns: ${ }^{5,6}$ 1) an outside pattern, in which an aneurysm rupture point is sealed from the outside by the clot; and 2) an inside pattern, in which the thrombus is attached to a rupture point from the inside of the aneurysm.
Computational fluid dynamics (CFD) uses patient-specific geometry models to characterize the pathophysiological mechanisms of aneurysm initiation, growth, and rupture. ${ }^{11}$ However, the relationships between hemodynamics and hemostatic patterns of ruptured aneurysms have not been investigated. It may be useful for neurosurgeons or interventional radiologists to know the hemostatic patterns before aneurysm obliteration, because hemostatic patterns may affect the treatment strategy. Thus, the goal of this study was to determine whether CFD analysis was useful to diagnose the hemostatic pattern of ruptured middle cerebral artery (MCA) aneurysms.

ABBREVATIONS ACA = anterior cerebral artery; $\mathrm{AFI}=$ aneurysm formation indicator; $\mathrm{CFD}=$ computational fluid dynamics; $\mathrm{GON}=$ gradient oscillatory number; $\mathrm{ICA}=$ internal cerebral artery; MCA = middle cerebral artery; OSI = oscillatory shear index; RIBBVA = Research Institute for Brain and Blood Vessels-Akita; STL = stereolithography; WSS = wall shear stress; WSSG $=$ WSS gradient.

SUBMITTED September 27, 2015. ACCEPTED March 8, 2016.

INCLUDE WHEN CITING Published online June 3, 2016; DOI: 10.3171/2016.3.JNS152264. 


\section{Methods}

This retrospective study was approved by the ethics committee of the Research Institute for Brain and Blood Vessels-Akita (RIBBVA). The patients from whom the aneurysms were obtained provided written informed consent for the use of their tissue and data.

\section{Patient Population and Study Protocol}

Between 2007 and 2011, 26 ruptured MCA aneurysms were clipped and harvested after 3D CT angiography at the RIBBVA. All aneurysms were located at the $\mathrm{M}_{1}$ bifurcation. One experienced neurosurgeon (T.I.) at the RIBBVA determined the hemostatic patterns of aneurysm rupture points, both at the time of surgery and at histopathological examination. Histopathological examination was performed under the direction of another experienced neuropathologist. Preoperative 3D CT angiography data were transferred to the Mie Chuo Medical Center, where experienced investigators who were unaware of the hemostatic patterns measured the morphological parameters and performed CFD analysis independently. The morphological and hemodynamic parameters were compared among the hemostatic patterns, and a determination was made as to whether CFD analysis was useful for the diagnosis or characterization of the hemostatic pattern.

\section{Histopathological Examination}

After the aneurysms were secured by neck clipping, the dome of the aneurysm, along with the rupture point, was very carefully dissected from surrounding structures with an irrigation system. The rupture point of the aneurysm was then visually inspected with regard to the mechanism of spontaneous hemostasis. Aneurysm domes were resected for histological analyses, while keeping the rupture point along with surrounding structures intact. The sections were subjected to hematoxylin and eosin staining to confirm the findings on visual inspection. Hemostatic patterns were divided into 4 different patterns depending on the location of clot formation: 1) outside pattern, when the surface of the aneurysm rupture point was sealed from the outside by the clot; 2) inside pattern, when the thrombus was attached to the rupture point from the inside of the aneurysm; 3) inside-outside pattern, when the thrombus extended from the inside to the outside of the rupture point; and 4) membrane pattern, when the rupture point was changed to a fibrin wall without distinct thrombus formation. ${ }^{6}$

\section{Morphological Parameters}

Morphological parameters were measured on stereolithographic models generated from preoperative 3D CT angiography studies using ImageJ software (National Institutes of Health). Neck width (NW) was defined as the maximum dimension at the neck orifice, aneurysm depth (AD) was defined as the aneurysm height perpendicular to the NW, and projection length (PL) was defined as the length between the middle point of NW and the farthest aneurysm surface point. Maximum size (MS) and parent artery diameter (PD) were documented at different views. Using these primary dimensions, shape indices were calculated. The aspect ratio (AR) was the ratio of $\mathrm{AD}$ to $\mathrm{NW}$, the projection ratio (PR) was the ratio of $\mathrm{PL}$ to $\mathrm{NW}$, and the size ratio (SR) was the ratio of MS to PD. ${ }^{14}$ In addition, neck area (NA), dome area (DA), and dome volume (DV) were measured with CFX-post (ANSYS CFX CFD15.0; ANSYS, Inc.). Volume-to-ostium area ratio (VOR) was defined as the ratio of DV to NA (Fig. 1). ${ }^{18}$

\section{CFD Analysis}

The patient-specific geometries were generated as stereolithography (STL) using the preoperative 3D CT angiography. Arterial lumen was segmented based on the intra-arterial CT value. The threshold CT value was determined at $40 \%$ between the maximum value in the extracranial internal carotid artery $\left(\mathrm{C}_{5}\right.$ segment $)$ and the minimum values in the extra-arterial area by the analytical software (Mimics 16.0, Materialise Japan). Vessels with diameters less than $1 \mathrm{~mm}$ were excluded from analysis, because it was judged that blood vessels with high Reynolds numbers had turbulent flow and were unsuitable for analysis of laminar flow (Magics 16.0, Materialise Japan). The STL was remeshed to improve the quality of the surface triangles (3-matics 6.0, Materialise Japan).

The computational hybrid meshes were generated with tetrahedral and prism elements (ANSYS ICEM CFD15.0, ANSYS, Inc.). Tetrahedral element sizes ranged from 0.1 to $0.6 \mathrm{~mm}$. Six prismatic boundary layers with a total thickness of $0.15 \mathrm{~mm}$ covered the vessel wall to locally ensure an accurate definition of the velocity gradient. The surface geometry was divided into the internal cerebral artery (ICA), anterior cerebral artery (ACA), parent artery $\left(\mathrm{M}_{1}\right.$ segment), branches $\left(\mathrm{M}_{2}\right.$ segment), and an aneurysm dome. A straight inlet extension was added to the $\mathrm{C}_{2}$ segment of the ICA to obtain fully developed laminar flow.

For the fluid domain, 3D incompressible laminar flow fields were obtained by solving the continuity and NavierStokes equations. Numeral modeling was performed using a commercially available CFD package (ANSYS CFX CFD15.0, ANSYS, Inc.). Blood was assumed to be an incompressible Newtonian fluid with a blood density of 1056 $\mathrm{kg} / \mathrm{m}^{3}$ and a blood dynamics viscosity of $0.0035 \mathrm{~Pa} \cdot \mathrm{sec}$. Typical flow waveform of phase-contrast MRI was scaled to achieve a physiological wall shear stress (WSS). ${ }^{3,13}$ Traction-free boundary conditions were applied at outlets of the MCA and ACA. The time steps were 0.0001 seconds, and 2 pulsatile cycles were taken as output.

WSS, WSS-related hemodynamic parameters at the dome, and invariant Q (vortex core region) were calculated as previously reported. . $^{2-10,12,17}$ WSS indicates the frictional force exerted by the flowing blood tangentially on the dome wall during the cardiac cycle. WSS-related hemodynamic parameters included 6 parameters. Normalized WSS (NWSS) was defined as the WSS ratio to the WSS magnitude of the parent artery, oscillatory shear index (OSI) was defined as the directional changes of WSS, WSS gradient (WSSG) was defined as the spatial gradient of WSS, gradient oscillatory number (GON) was defined as fluctuations in the WSS gradient integrated over 1 cardiac cycle, and aneurysm formation indicator (AFI) was defined as an index to detect the flow stagnation zones. Invariant $\mathrm{Q}$ identified zones of swirling blood flow motion in 

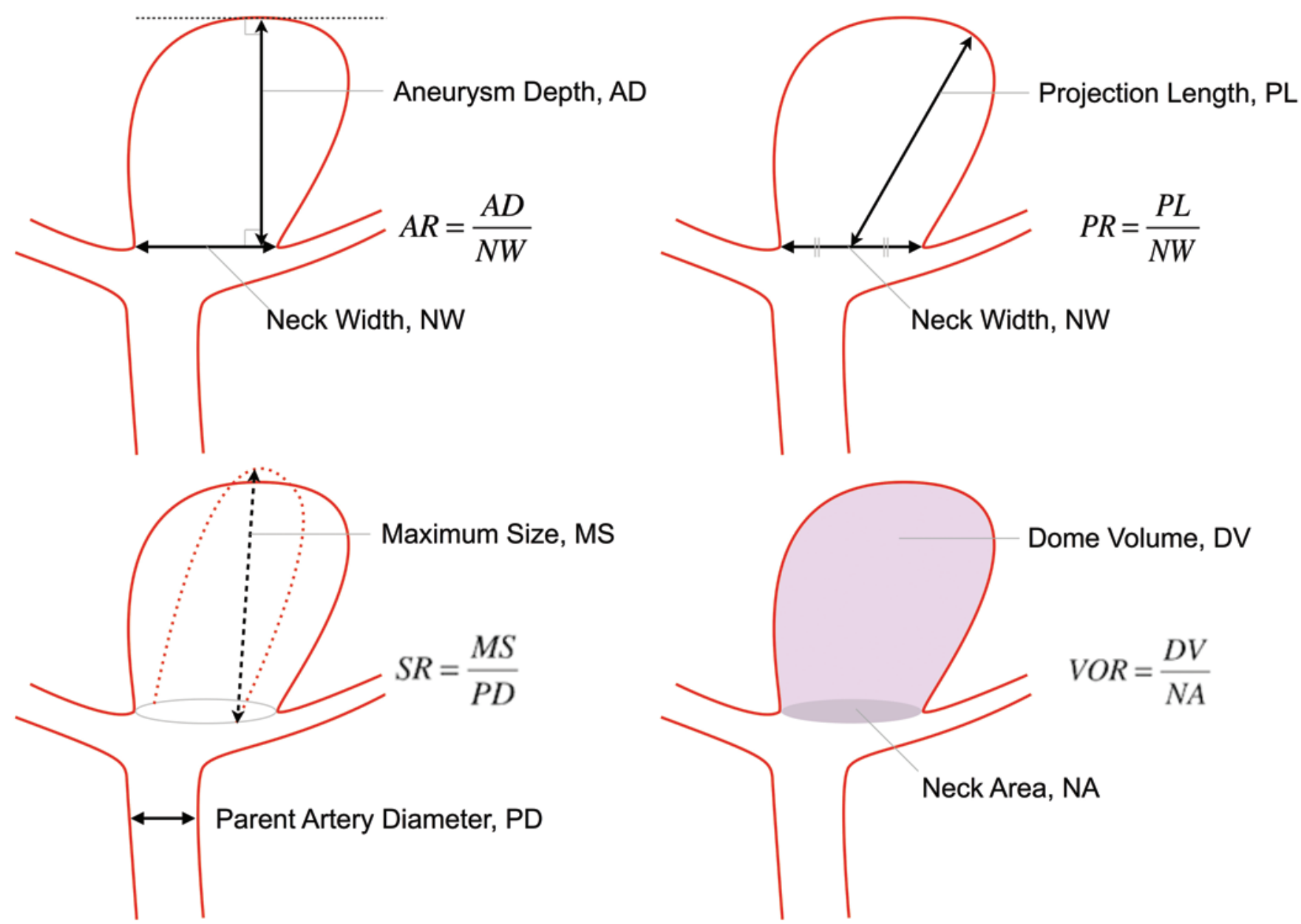

FIG. 1. Schema of aneurysm dimensions and shape indices. $A R=$ aspect ratio; $P R=$ projection ratio; $S R=$ size ratio; $V O R=$ volume-to-ostium area ratio.

the aneurysm dome during the cardiac cycle. AFI was calculated at the systolic phase. Other data were time average values over the second pulsatile cycle of flow simulation.

\section{Statistical Analysis}

All values are expressed as the median (interquartile range [IQR]). Statistical analyses were performed using SPSS software (version 22.0, IBM Corp.). A Mann-Whitney U-test was performed to assess statistical significance in difference between the inside and the outside patterns. A p value less than 0.05 was considered to indicate statistical significance.

\section{Results}

\section{Patient Population and Histopathological Examinations}

The median patient age was 62.5 years (IQR 54.8-67.8 years), and the patient group included 4 men and 22 women. The median value for the aneurysm maximum diameter was $6.18 \mathrm{~mm}$ (IQR 2.86-13.18 mm). Clinical characteristics of patients with a ruptured MCA aneurysm and the hemostatic patterns are listed in Table 1. Among 26 aneurysms, 3 aneurysms (1 inside pattern, 1 outside pat- tern, and 1 inside-outside pattern) were excluded because their geometry models could not be generated due to the low vascular CT values. As a result, we analyzed 23 aneurysms; 12 had inside patterns, 8 had outside patterns, 2 had inside-outside patterns, and 1 had a membrane pattern. Because the number of aneurysms with inside-outside and membrane patterns was too small, we combined them into an "other" pattern category and investigated the morpho-

TABLE 1. Patient characteristics and aneurysm hemostatic patterns in 26 cases

\begin{tabular}{lccc}
\hline & \multicolumn{3}{c}{ Hemostatic Pattern } \\
\cline { 2 - 4 } Characteristic & Inside $(\mathrm{n}=13)$ & Outside $(\mathrm{n}=9)$ & Other $(\mathrm{n}=4)$ \\
\hline Patient age, yrs & \multicolumn{3}{c}{64.0} \\
\hline Median & 62.0 & 63.0 & $59.8-69.0$ \\
\hline IQR & $54.0-68.0$ & $57.0-66.0$ & 1 \\
\hline Sex, no. of patients & & & 3 \\
\hline Male & 2 & 1 &
\end{tabular}

$\mathrm{IQR}=$ interquartile range. 
TABLE 2. Summary of morphological parameters in 23 aneurysms*

\begin{tabular}{lcccc}
\hline & & \multicolumn{3}{c}{ Hemostatic Pattern } \\
\cline { 2 - 4 } Parameter & Inside $(\mathrm{n}=12)$ & Outside $(\mathrm{n}=8)$ & Other $(\mathrm{n}=3)$ & $\mathrm{p}$ Value† \\
\hline Aneurysm depth, $\mathrm{mm}$ & $3.73(3.28-4.76)$ & $5.97(4.60-7.33)$ & $6.19(5.68-6.99)$ & 0.037 \\
\hline Projection length, $\mathrm{mm}$ & $4.41(3.33-5.18)$ & $5.97(4.74-8.95)$ & $7.36(7.32-7.58)$ & 0.031 \\
\hline Max size, $\mathrm{mm}$ & $5.45(4.53-6.11)$ & $8.41(5.38-10.53)$ & $8.70(8.24-10.85)$ & 0.045 \\
\hline Neck width, $\mathrm{mm}$ & $4.39(3.21-4.91)$ & $5.44(3.69-6.43)$ & $7.01(5.22-7.37)$ & 0.132 \\
\hline Aspect ratio & $0.88(0.73-0.97)$ & $1.09(0.83-1.51)$ & $0.88(0.78-1.58)$ & 0.203 \\
\hline Projection ratio & $0.91(0.85-1.09)$ & $1.12(0.90-1.80)$ & $1.04(1.00-1.66)$ & 0.113 \\
\hline Size ratio & $2.81(1.78-3.00)$ & $4.35(2.17-5.19)$ & $3.74(3.28-5.64)$ & 0.082 \\
\hline Neck area, $\mathrm{cm}^{2}$ & $0.13(0.07-0.15)$ & $0.21(0.10-0.27)$ & $0.20(0.14-0.25)$ & 0.090 \\
\hline Dome area, $\mathrm{cm}^{2}$ & $0.48(0.31-0.63)$ & $1.31(0.80-1.82)$ & $0.86(0.83-1.35)$ & 0.011 \\
\hline Dome volume, $\mathrm{cm}^{3}$ & $0.04(0.02-0.05)$ & $0.12(0.08-0.23)$ & $0.65(0.64-1.29)$ & 0.014 \\
\hline VOR, mm & $3.21(2.08-4.05)$ & $5.95(3.97-10.69)$ & $6.79(4.96-7.25)$ \\
\hline
\end{tabular}

VOR = volume-to-ostium area ratio.

* Data are presented as median (IQR). Three aneurysms were excluded from analysis because their geometry models could not be generated due to the low vascular CT values.

† Mann-Whitney U-test between inside and outside patterns.

logical and hemodynamic differences between the inside and outside patterns.

\section{Morphological Parameters}

Morphological parameters are shown in Table 2. Inside-pattern aneurysms had significantly lower aneurysm depth, lower projection length, lower maximum size, lower dome area, lower dome volume, and lower VOR than outside-pattern aneurysms (median $3.73 \mathrm{~mm}$ vs $5.97 \mathrm{~mm}$, $\mathrm{p}=0.037 ; 4.41 \mathrm{~mm}$ vs $5.97 \mathrm{~mm}, \mathrm{p}=0.031 ; 5.45 \mathrm{~mm}$ vs $8.41 \mathrm{~mm}, \mathrm{p}=0.045 ; 0.48 \mathrm{~cm}^{2}$ vs $1.31 \mathrm{~cm}^{2}, \mathrm{p}=0.011 ; 0.04$ $\mathrm{cm}^{3}$ vs $0.12 \mathrm{~cm}^{3}, \mathrm{p}=0.014 ; 3.21 \mathrm{~mm}$ vs $5.95 \mathrm{~mm}, \mathrm{p}=0.014$, respectively).

\section{Hemodynamic Parameters}

Hemodynamic parameters are shown in Table 3. Distributions of WSS, OSI, WSSG, GON, AFI, and invariant $\mathrm{Q}$ for the inside and outside patterns are shown in Fig. 2. The inside pattern had significantly lower GON, higher AFI, and lower invariant Q than the outside pattern (median 0.0234 vs $0.0289, \mathrm{p}=0.023 ; 0.986$ vs 0.963 , $\mathrm{p}=0.041 ;-0.80110^{-2} / \mathrm{sec}^{2} \mathrm{vs}-0.12410^{-2} / \mathrm{sec}^{2}, \mathrm{p}=0.045$, respectively).

\section{Discussion}

Our results demonstrated that aneurysm hemostatic patterns could be distinguished by the morphological and hemodynamic parameters. The blood flow in inside-pattern aneurysms had characteristic hemodynamic features, such as higher AFI, lower GON, and lower invariant Q, meaning less flow stagnation and simpler flow patterns. The shape indices of inside-pattern aneurysms were smaller. A possible explanation for this might be related to a thrombus or platelet attaching to the rupture point from the inside, causing the inside-pattern aneurysm to become smaller after rupture than before rupture. Another possible reason is that the blood flow of the inside pattern aneurysms may become stable through the transformation

TABLE 3. Summary of hemodynamic parameters in 23 aneurysms ${ }^{*}$

\begin{tabular}{|c|c|c|c|c|}
\hline \multirow[b]{2}{*}{ Parameter } & \multicolumn{3}{|c|}{ Hemostatic Pattern } & \multirow[b]{2}{*}{$p$ Value } \\
\hline & Inside $(\mathrm{n}=12)$ & Outside $(n=8)$ & Other $(n=3)$ & \\
\hline WSS, $\mathrm{Pa}$ & $0.528(0.311-1.293)$ & $0.441(0.225-0.572)$ & $0.257(0.156-2.294)$ & 0.440 \\
\hline NWSS & $0.184(0.136-0.344)$ & $0.176(0.086-0.215)$ & $0.165(0.0952-0.293)$ & 0.589 \\
\hline OSI & $0.0120(0.0059-0.0193)$ & $0.0173(0.0126-0.0310)$ & $0.0315(0.0198-0.0366)$ & 0.316 \\
\hline WSSG, $\mathrm{Pa} / \mathrm{mm}$ & $0.666(0.549-1.230)$ & $0.555(0.351-0.799)$ & $0.384(0.232-2.137)$ & 0.247 \\
\hline GON & $0.0234(0.0131-0.0285)$ & $0.0289(0.0243-0.0365)$ & $0.0288(0.0235-0.0300)$ & 0.023 \\
\hline AFI & $0.986(0.973-0.9848)$ & $0.963(0.956-0.982)$ & $0.990(0.974-0.990)$ & 0.041 \\
\hline Invariant $\mathrm{Q}, 10^{-2} / \mathrm{sec}^{2}$ & $-0.801(-3.633$ to -0.409$)$ & $-0.124(-0.454$ to -0.084$)$ & $-0.446(-3.33$ to -0.250$)$ & 0.045 \\
\hline
\end{tabular}

$\mathrm{AFI}=$ aneurysm formation indicator; GON = gradient oscillatory number; NWSS = normalized wall shear stress; OSI = oscillatory shear index; WSS = wall shear stress; WSSG = wall shear stress gradient .

* Data are presented as median (IQR).

$\dagger$ Mann-Whitney U-test between inside and outside patterns. 
A

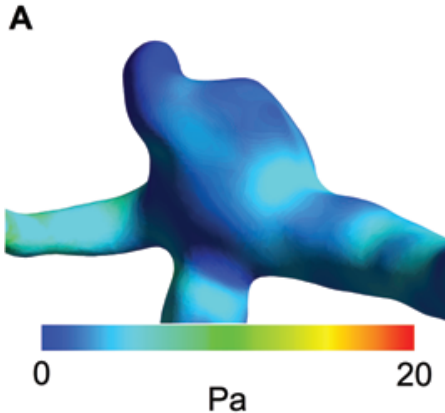

D

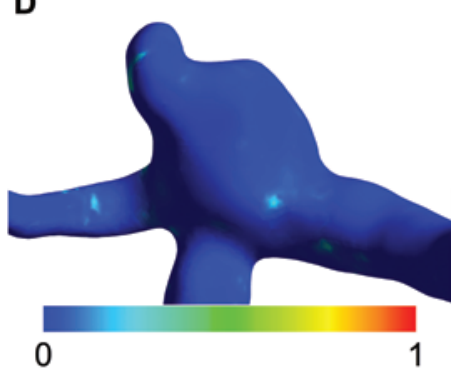

G

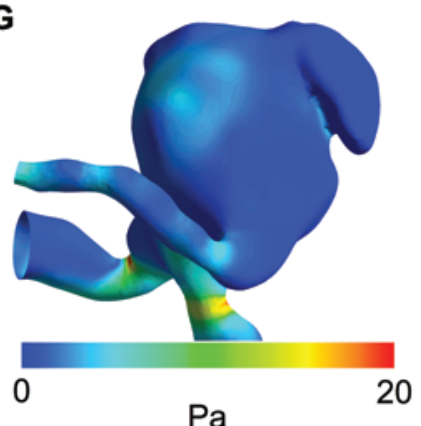

$\mathrm{Pa}$

J

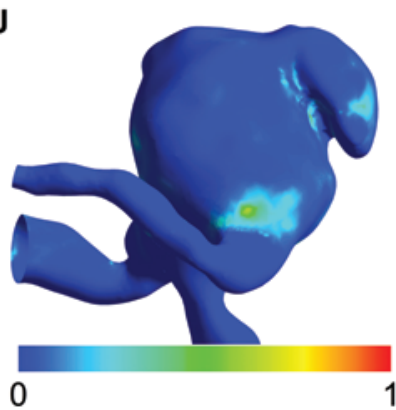

B

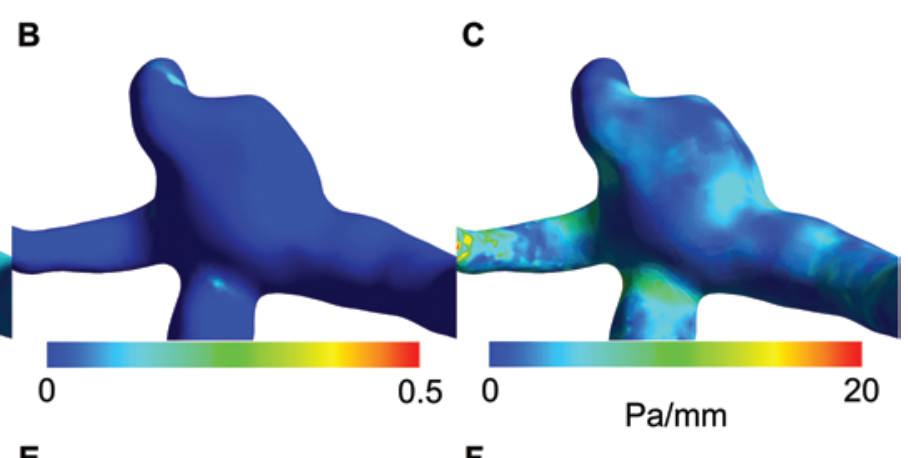

E

F
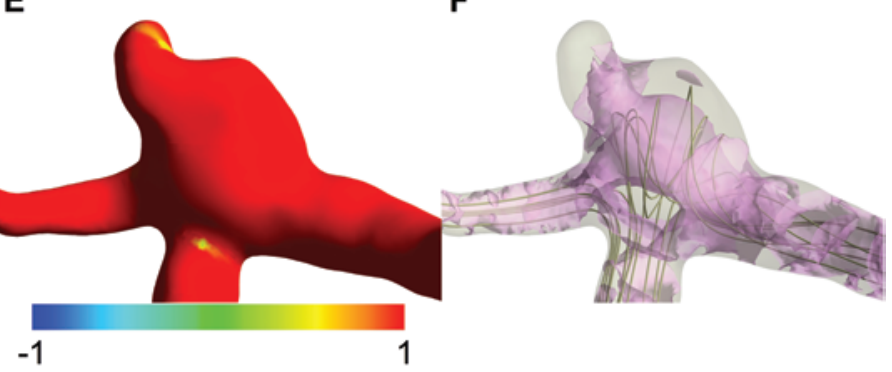

H

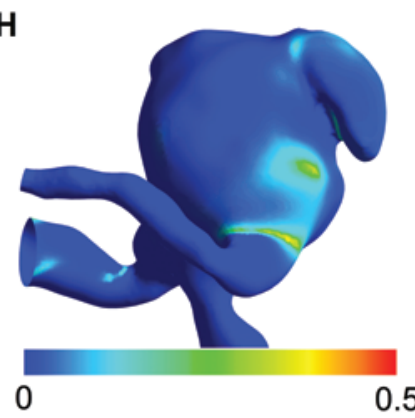

$\mathbf{K}$

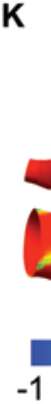

I

0.5

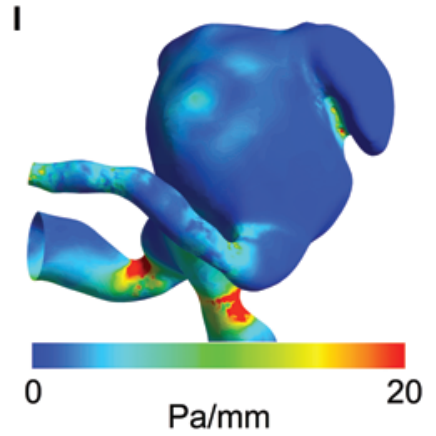

L

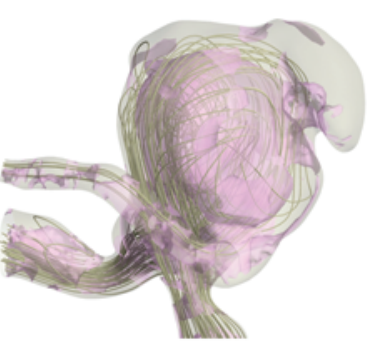

FIG. 2. Visualization of hemodynamic parameters on computational fluid dynamics analyses of representative cases of insidepattern $(\mathbf{A}-\mathbf{F})$ and outside-pattern $(\mathbf{G}-\mathbf{L})$ aneurysms showing wall shear stress (A and $G)$, oscillatory shear index $(B$ and $H)$, wall shear stress gradient ( $C$ and $I)$, gradient oscillatory number $(D$ and $J)$, aneurysm formation indicator $(E$ and $K)$, and invariant $Q(F$ and $L$, isosurface of invariant $Q$ above 0 and $3 D$ streamlines).

of the lesion to a regular smooth shape due to thrombus formation into the aneurysm dome.

AFI is a parameter to detect a flow stagnation zone. ${ }^{8}$ Inside-pattern aneurysms had significantly higher AFI and therefore would have a region of less irregular fluid domain (i.e., less stagnation).

Invariant $\mathrm{Q}$ is based on the local velocity gradient tensor and identifies zones of swirling motion. ${ }^{2}$ A high invariant $\mathrm{Q}$ means that the proportion of the aneurysm occupied by the whirlpool domain is high. A recent study reported that "the aneurysm flow structure is drastically different from systole to diastole and is dominated by the motion of highly dynamic vortex core" in the dome, emphasizing the importance of vortex formation phenomena. This study showed that inside patterns had significantly lower invariant $\mathrm{Q}$ and suggested that detection of a simpler flow pattern is potentially useful to diagnose inside-pattern aneurysms. 
GON, which measures fluctuations of the WSS gradient integrated over 1 cardiac cycle, is a hemodynamic parameter developed to detect the initiation of cerebral aneurysms. ${ }^{15} \mathrm{GON}$ was also described as a parameter to quantify the degree of oscillating tension/compression forces on endothelial cells. ${ }^{1,4}$ A previous report showed that there was no significant difference in GON between ruptured and unruptured aneurysm. ${ }^{12}$ Elevated GON correlates with regions of aneurysm formation, and GON is relevant to disturbed flow. ${ }^{15}$ Because the surface of the aneurysm rupture point was sealed from the outside by a platelet plug or fibrin net, outside-pattern aneurysms would retain an irregular shape after aneurysm rupture. As a result, outside-pattern aneurysms could have significantly higher GON, which indicates that outside-pattern aneurysms would have a larger flow turbulence domain. Thus, GON might be a useful parameter to characterize the hemostatic pattern.

To our knowledge, no study has shown the relevance of hemostatic patterns of ruptured cerebral aneurysms to hemodynamic characteristics. However, the geometrical measurements might be affected by thrombus formation. That is, the geometrical characteristics of inside-pattern aneurysms might largely differ from those of the aneurysms before rupture, and therefore hemodynamic parameters might also significantly change after aneurysm rupture due to intra-aneurysmal thrombus. Indeed, results from the present study suggest that hemodynamic parameters analyzed via CFD were as important as morphological parameters in predicting the hemostatic pattern. Therefore, changes in the aneurysm geometry in association with hemostasis of ruptured aneurysms would have an influence on the hemodynamics. However, as it was impossible to visualize or quantify the intra-aneurysmal thrombus in this study, we could not determine how intraaneurysmal thrombus affected the hemodynamic patterns. In this regard, further studies are needed.

It may be useful to determine the hemostatic patterns of ruptured cerebral aneurysms before treatment. For example, a ruptured aneurysm with the outside hemostatic pattern may have higher risks for premature aneurysm rupture during surgical clip placement. However, if neurosurgeons know the finding before the operation, they can take care not to exfoliate the clot on the rupture point or use a temporary clip before dissecting the aneurysm dome from the surrounding tissues, thereby preventing premature aneurysm rupture. Information pertaining to the inside-pattern aneurysms may be useful for interventional neuroradiologists or endovascular neurosurgeons. In inside-pattern aneurysms, the actual aneurysm shape may differ from the shape observed on angiography, and the intra-aneurysmal thrombus may carry higher risks for aneurysm recurrence after coil embolization, thus requiring more meticulous imaging follow-up. In inside-pattern aneurysms, seemingly sufficient packing may not preclude reopening of the aneurysm, because factors other than coil compaction, including intra-aneurysmal thrombus resolution, migration of the coil mesh into the thrombus, or both, can cause the aneurysm to recanalize over time..$^{16}$

There are some limitations to this study. First, patientspecific geometry was generated by a method dependent on CT value measurement; because it was impossible to generate the geometry in patients with severe subarachnoid hemorrhage, 3 aneurysms had to be excluded, which might have influenced the findings of the study. Second, all MCA aneurysms are not configured in the same manner. There are several other morphological and peri-aneurysmal environmental differences, such as the relationships to adjacent branches, adjacent branch diameter, and the relationship of the in-flow zone to the $\mathrm{M}_{1}$ segment, that may have influenced hemostasis. Third, this study included only MCA aneurysms, in order to exclude the possibility that the location of aneurysm may affect the findings. Lastly, the aneurysms came from a single center, and the number of cases was small. Further large-scale studies with aneurysms in different locations in the cerebral vasculature are required to verify the present findings.

\section{Conclusions}

Inside-pattern aneurysms may have simpler flow patterns and less flow stagnation when compared with outside pattern aneurysms. CFD may be useful to characterize the hemostatic pattern of ruptured cerebral aneurysms.

\section{Acknowledgments}

We thank Hajime Miyata, MD, from the Department of Neuropathology, Research Institute for Brain and Blood Vessels, Akita, Akita, Japan, for his help with the histopathological data collection.

\section{References}

1. Chen H, Selimovic A, Thompson H, Chiarini A, Penrose J, Ventikos Y, et al: Investigating the influence of haemodynamic stimuli on intracranial aneurysm inception. Ann Biomed Eng 41:1492-1504, 2013

2. Chong MS, Perry AE, Cantwell BJ: A general classification of three-dimensional flow fields. Phys Fluids 2:765-777, 1990

3. Ford MD, Alperin N, Lee SH, Holdsworth DW, Steinman DA: Characterization of volumetric flow rate waveforms in the normal internal carotid and vertebral arteries. Physiol Meas 26:477-488, 2005

4. Ford MD, Hoi Y, Piccinelli M, Antiga L, Steinman DA: An objective approach to digital removal of saccular aneurysms: technique and applications. Br J Radiol 82:S55-S61, 2009

5. Ishikawa T, Nakayama N, Yoshimoto T, Aoki T, Terasaka S, Nomura M, et al: How does spontaneous hemostasis occur in ruptured cerebral aneurysms? Preliminary investigation on 247 clipping surgeries. Surg Neurol 66:269-276, 2006

6. Ishikawa T, Miyata H, Moroi J, Hikichi K, Yoshioka S, Kobayashi S, et al: Pathological consideration for hemostatic clot on ruptured cerebral aneurysms. Surg Cereb Stroke (Jpn) 40:223-228, 2012

7. Le TB, Troolin DR, Amatya D, Longmire EK, Sotiropoulos F: Vortex phenomena in sidewall aneurysm hemodynamics: experiment and numerical simulation. Ann Biomed Eng 41:2157-2170, 2013

8. Lei M, Kleinstreuer C, Truskey GA: A focal stress gradientdependent mass transfer mechanism for atherogenesis in branching arteries. Med Eng Phys 18:326-332, 1996

9. Malek AM, Alper SL, Izumo S: Hemodynamic shear stress and its role in atherosclerosis. JAMA 282:2035-2042, 1999

10. Mantha A, Karmonik C, Benndorf G, Strother C, Metcalfe R: Hemodynamics in a cerebral artery before and after the formation of an aneurysm. AJNR Am J Neuroradiol 27:1113-1118, 2006 
11. Meng H, Tutino VM, Xiang J, Siddiqui A: High WSS or low WSS? Complex interactions of hemodynamics with intracranial aneurysm initiation, growth, and rupture: toward a unifying hypothesis. AJNR Am J Neuroradiol 35:1254-1262, 2014

12. Miura Y, Ishida F, Umeda Y, Tanemura H, Suzuki H, Matsushima S, et al: Low wall shear stress is independently associated with the rupture status of middle cerebral artery aneurysms. Stroke 44:519-521, 2013

13. Murray CD: The physical principle of minimum work applied to the angle of branching of arteries. J Gen Physiol 9:835-841, 1926

14. Rahman M, Smietana J, Hauck E, Hoh B, Hopkins N, Siddiqui A, et al: Size ratio correlates with intracranial aneurysm rupture status: a prospective study. Stroke 41:916-920, 2010

15. Shimogonya $Y$, Ishikawa T, Imai Y, Matsuki N, Yamaguchi $\mathrm{T}$ : Can temporal fluctuation in spatial wall shear stress gradient initiate a cerebral aneurysm? A proposed novel hemodynamic index, the gradient oscillatory number (GON). J Biomech 42:550-554, 2009

16. Sluzewski M, van Rooij WJ, Slob MJ, Bescós JO, Slump CH, Wijnalda D: Relation between aneurysm volume, packing, and compaction in 145 cerebral aneurysms treated with coils. Radiology 231:653-658, 2004

17. Taylor CA, Hughes TJ, Zarins CK: Finite element modeling of three-dimensional pulsatile flow in the abdominal aorta: relevance to atherosclerosis. Ann Biomed Eng 26:975-987, 1998

18. Yasuda R, Strother CM, Taki W, Shinki K, Royalty K, Pulfer $\mathrm{K}$, et al: Aneurysm volume-to-ostium area ratio: a parameter useful for discriminating the rupture status of intracranial aneurysms. Neurosurgery 68:310-318, 2011

\section{Disclosures}

The authors report no conflict of interest concerning the materials or methods used in this study or the findings specified in this paper.

\section{Author Contributions}

Conception and design: Ishikawa, Ishida. Acquisition of data: Ishikawa. Analysis and interpretation of data: Tsuji, Ishida. Critically revising the article: Ishida, Suzuki. Reviewed submitted version of manuscript: Ishida, Furukawa, Miura, Shiba, Sano, Tanemura, Umeda, Shimosaka, Suzuki. Approved the final version of the manuscript on behalf of all authors: Tsuji. Statistical analysis: Tsuji. Administrative/technical/material support: Ishida.

\section{Correspondence}

Masanori Tsuji, Department of Neurosurgery, Mie University Graduate School of Medicine, 2-174 Edobashi, Tsu, Mie 5148507, Japan. email: masanori00010413@icloud.com. 